\title{
Should practitioners promote physical activity as a treatment for depression?
}

\author{
${ }^{1} \mathrm{M}$ Donaghy, ${ }^{2} \mathrm{AH}$ Taylor \\ ${ }^{1}$ Dean, School of Health Sciences, Queen Margaret University, Edinburgh; ${ }^{2}$ Professor in Exercise and Health Psychology, School of Sport $\mathcal{E}$ \\ Health Sciences, University of Exeter, UK
}

\begin{abstract}
For many years, experts have been debating the pros and cons of exercise for depression. Proponents of exercise for depression point to those clinical trials which have shown that exercise improves mood, while sceptics point out the methodological problems in many of the apparently positive trials, and the uncertainties around the acceptability of exercise as a treatment for depression. Here two experts critically review the evidence around exercise for depression, provide arguments for and against the promotion of physical activity as a treatment for depression, explore issues around the generalisability of exercise as a treatment for depression and look to the future by discussing ongoing trials that will provide more evidence to inform this important debate.
\end{abstract}

KEYWORDS Depression, exercise, physical activity, stepped care

DECLARATION OF INTERESTS Professor Taylor has been involved with the Trial of Exercise and Depression (TREAD), which receives funding from HTA (NIHR).

\author{
Correspondence to \\ M Donaghy, \\ School of Health Sciences, \\ Queen Margaret University, Edinburgh, \\ Musselburgh EH2 I 6UU, UK \\ tel+ 44 (0) I3I 4740563 \\ email mdonaghy@qmu.ac.uk \\ AH Taylor, \\ School of Sport \& Health Sciences, \\ Richards Building, St Luke's Campus, \\ University of Exeter, \\ Exeter EXI 2LU, UK \\ tel +44 (0) I392 72474 \\ email a.h.taylor@exeter.ac.uk
}

\section{Depression: a major cause of morbidity and disability}

Depression is not only a major cause of disability' but also has indirect effects on physical morbidity, through both biological (such as stress reactivity and hypertension) and behavioural processes (such as adverse effects on smoking, diet and physical inactivity). ${ }^{2}$ There has been a dramatic increase in prescribing antidepressant medication in primary care in the past 10-20 years, and while traditional psychotherapies (for example, cognitive behavioural therapy) may be more acceptable to patients than antidepressants, access to these treatments may sometimes be limited. ${ }^{3}$ Within a stepped care framework, more recent interest has focused on low-intensity interventions (such as counselling, exercise programmes and self-management/help approaches) for the treatment of mild to moderate depression. ${ }^{4}$ The stepped care framework provides a guide for commissioners and practitioners to identify patients with lesser needs for mental health care and provide low-intensity support and increase access to psychological therapies. Exercise treatments for depression have not typically been included as a 'psychological therapy', but this article considers an approach to promoting physical activity through counselling.

The primary aim of this article is to offer informed debate about the role of physical activity and exercise as an emerging therapy. It begins with a review of research evidence for effectiveness and the recent National Institute for Health and Clinical Excellence (NICE) guidelines for the treatment of depression. We then consider the pros and cons of exercise and physical activity as a therapy, both with this evidence base in mind and in consideration of other literature and emerging initiatives. We will explore key issues around physical activity for depression, including patient acceptability and the availability of suitable exercise programmes for people with depression.

The evidence base for exercise as a therapy for depression: what do trials and guidelines say?

Numerous reviews on the effectiveness of exercise for reducing depression have been conducted, but we will focus on the two most recent that focused on treating clinical depression. The first was a systematic review that included 28 randomised controlled trials (RCTs), 25 of which were subjected to meta-analysis, and compared exercise with standard treatment, no treatment or a placebo treatment. ${ }^{5}$ The 23 trials $(n=907)$ which compared exercise to a placebo or a control intervention found the pooled standardised mean difference (SMD) in symptom severity post-treatment was $-0.82 \quad(95 \%$ confidence interval $[\mathrm{Cl}]-|| 2,.-0.5 \mathrm{I})$, indicating a large treatment effect. However, only three studies $(n=2 \mid 6)$ met more stringent methodological quality (e.g.allocation concealment, intention-to-treat analysis and blind assessment), and the pooled SMD for these studies was only $-0.42(95 \% \mathrm{Cl}-0.88,0.03)$ (i.e. a non-significant moderate treatment effect).

Other reviews have also reported that exercise improved depressive symptoms. ${ }^{1,6}$ The 25 studies reviewed for the NICE 2009 guidelines on depression led to the following recommendation:

Physical activity programmes for people with persistent sub-threshold depressive symptoms or mild to moderate depression should: 
- be delivered in groups with support from a competent practitioner;

- consist typically of three sessions per week of moderate duration (45-60 minutes) over 10-14 weeks (average 12 weeks).

Similarly the Scottish Intercollegiate Guidelines Network $\left(\right.$ SIGN) ${ }^{6} 2010$ guidelines on non-pharmacological treatment of depression recommend that structured exercise may be considered as a treatment option for patients with depression. However, the guidelines are less prescriptive, favouring an exercise-counselling approach recommending that individuals who are interested in using exercise as a treatment intervention for depression should 'be referred to appropriate exercise counselling and activities in their local community that are relevant to the type of exercise they feel they will enjoy'.

In summary, the purist may argue that there is insufficient evidence from randomised controlled trials of stringent methodological quality to promote exercise as a therapy for treating depression, whereas a less conservative assessment may lead to a practitioner following the NICE or SIGN guidelines. We will now identify the pros and cons for promoting exercise as a therapy for depression, before adding further discussion to the debate.

There is insufficient evidence to recommend physical activity as an intervention

Historically, there has been insufficient investment in rigorous trials on the effects of exercise as a treatment for depression. Among the studies included in the Cochrane review, ${ }^{5}$ only three studies involved randomising more than 50 participants into an exercise intervention. Studies typically involve research volunteers who are likely to be less depressed than patients seeking medical intervention, and therefore more motivated and less inclined to drop out of an exercise intervention. Indeed, interventions have mostly involved structured exercise programmes, with doses of exercise designed to enhance fitness, and therefore fail to attract those less interested in formal exercise sessions. Such programmes, in the form of general practitioner (GP) exercise referral schemes in the UK, have shown little effect on changing long-term levels of physical activity and would seem unlikely to reduce depression in a sustainable way. ${ }^{7,8}$ Most exercise and depression studies used self-reported questionnaires to assess depression, with few using a structured clinical interview. Without the structured clinical interview it is not possible to confirm whether the majority of people participating in these trials met the diagnostic criteria for clinical depression.

The wider applicability of exercise for people with clinical depression is also questionable. We do not know if exercise is a cost-effective treatment, nor do we know the percentage uptake of people with depression who are offered an exercise referral. The barriers researched in a few pragmatic studies suggest low motivation, pessimism, physical inactivity, low self efficacy and withdrawal may limit exercise as a treatment of choice for people with clinical depression. ${ }^{910}$ Finally, exercise interventions often involve social contact and it is hard to separate out the effects of exercise versus social interaction. In summary, we know little about how actual patients would respond to an exercise programme as a treatment for depression.

There is sufficient evidence for physical activity as a therapy for depression

As the above reviews and many others reveal, the majority of trials undertaken in the past ten years indicate that exercise is effective in the treatment of mild to moderate depression. 1,5 The fact that these findings, with moderate to large effect sizes, are from studies with relatively small sample sizes reinforces the potential effect in clinical practice. It may be rather unjust to critique studies on the basis of some methodological standards normally associated with pharmacological trials, such as allocation concealment and blinded assessment. Although the three trials reported in the Cochrane review that met the most stringent methodological criteria revealed no significant effect of exercise, the SMD of -0.423 was greater than the effect sizes reported in two recent meta-analyses of controlled trials of antidepressants from the US Food and Drug Administration (SMD=0.32 and SMD $=0.31) .^{11,12}$

The same investment in large-scale exercise trials to investigate the effects on depression would very likely reveal significant effects. Moreover, participants are very aware that they are in an exercise condition and may inadvertently talk about this during follow-up assessments. When participants have been randomised to a plausible placebo condition the treatment often serves to dilute any effects of exercise, simply because a low-intensity exercise class, such as stretching, can itself produce a therapeutic effect. Participants in a control condition invariably increase their physical activity, simply because they have volunteered for a study to test the effects of exercise and this resolves their disappointment in not receiving the active intervention.

If there is any doubt that exercise has causal effects on reducing depression it is worthwhile considering the criteria for causality referred to by Hill, ${ }^{13}$ and considered in the present context by Taylor and Faulkner. ${ }^{14}$ Certainly there is a consistently moderate to strong effect in the association between exercise and depression across age groups and for males and females in both cross-sectional and prospective studies, there is support for a doseresponse relationship with more physical activity having a greater effect on depression ${ }^{15}$ and animal and human models support biological plausibility, although the precise mechanisms remain uncertain. ${ }^{16}$

Finally, it is worth mentioning that exercise interventions, compared with antidepressant treatments, are associated 
with few adverse events. ${ }^{5}$ In summary, there appears to be sufficient evidence that physical activity as an intervention should be recommended.

Consideration of the pros and cons for recommending physical activity as a therapy for depression raises a number of important issues such as the following: is exercise an acceptable therapy for patients and what types of support are preferred (if different from the NICE guidelines)? Can GPs confidently refer patients to competent practitioners (mentioned in the NICE guidelines)?

Is exercise an acceptable therapy for patients with depression and, if so, what types of support are preferred?

Yes: exercise is widely acceptable as a treatment for depression Several consumer surveys have examined whether exercise is an acceptable patient therapy for reducing depression. The mental health charity Mind ${ }^{17}$ found that $83 \%$ of people with mental health problems looked to exercise to lift their mood or to reduce stress, with two thirds indicating that it helped to relieve their depression. In a web-based survey of 420 people treated for depression, ${ }^{18} 71 \%$ reported that they would like more information or help with increasing physical activity as a therapy, with more interest $(\mathbf{7 3 \%})$ in an exercise-referral scheme (i.e. at an exercise facility) than a walking group (51\%). Females were considerably more interested in all forms of exercise therapy than males. Interestingly, only $52 \%$ reported that they had been encouraged to increase physical activity as part of their treatment, revealing a discrepancy between need and provision. Surveys by the Mental Health Foundation revealed an increased interest in exercise therapy between 2005 and 2009. ${ }^{77,19}$

No: exercise is not widely acceptable as a treatment for depression

Although the surveys quoted above suggest that patients with depression are willing to participate in exercise, the evidence to date from GP exercise referral schemes indicates that take-up and drop-out rates are highly variable. ${ }^{7,8}$ Reasons include exercise being regarded as a low-priority treatment option by some GPs, lack of access to facilities, low motivation, lack of social support and time commitment required by participants. ${ }^{20}$ More information on GP access to, and the proportion of eligible patients using, exercise counselling, exercise referral schemes and community leisure facilities is required to inform the debate about the generalisability of exercise as a treatment for depression.

Can GPs confidently refer patients to competent practitioners (mentioned in the NICE guidelines)?

General practitioner exercise referral schemes ${ }^{18}$ allow people with mental health problems to access exercise facilities in their local community. The Mental Health Foundation-commissioned report Up and running! revealed that only $5 \%$ of the GPs surveyed consider exercise as one of their three most common treatment responses ${ }^{17}$ (compared with $92 \%$ who would consider using medication), although there was an increase between 2005 and 2009. The 2009 report Moving on up also revealed that less than half of GPs surveyed had access to an exercise referral scheme. ${ }^{19}$ In addition to availability of a referral scheme, GPs can have some concerns about the suitability of structured exercise programmes for clients with low self-esteem and social physique anxiety, and the competencies of exercise practitioners. ${ }^{20}$ Very few have received any training to work with patients with mental health problems. If health professionals have concerns about the qualifications of an exercise practitioner then they can gain further information from the Register for Exercise Professionals (www.exerciseregister.org), set up by the government to raise the standards in the fitness industry, at the same time as the launch of the National Quality Assurance Framework for Exercise Referral Schemes. ${ }^{21}$

\section{Uncertainties about the optimum 'dose' of exercise}

The NICE guidelines promote a fixed dose of structured exercise, largely because most trials have adopted this approach, and there has been a belief that fitness gain was critical to reducing depression. However, recent research suggests that promoting an equivalent to the public health dose is also effective in reducing depression, but that frequency does not matter. ${ }^{15}$ This study compared frequency of exercise (three or five days per week) and total energy expenditure per week (7 kcal/kg/ week 'low dose' versus $17.5 \mathrm{kcal} / \mathrm{kg} /$ week 'public health dose') in a 12-week protocol. Four aerobic exercise conditions allowed these comparisons; two groups exercised on three days a week - one expended $7 \mathrm{kcal} /$ $\mathrm{kg} /$ week and the other $17.5 \mathrm{kcal} / \mathrm{kg} /$ week; two other groups exercised on five days a week but expended the same totals of either 7 or $17.5 \mathrm{kcal} / \mathrm{kg} /$ week. Participants were randomly assigned to one of these four groups or to a placebo exercise condition which involved stretching exercises on three days of the week. In summary this means that depression can be reduced with $3 \times 10$ minutes or $2 \times 15$ minutes of brisk walking a day. This is an important study as this level of physical activity may be more readily integrated into the daily routine of people living with depression.

There is also scope for other professionals to promote physical activity as a therapy who may be less competent in developing a structured programme in an exercise facility.

\section{The future}

A large UK trial (TREAD) is due to report its findings in $201 \mathrm{I}$ on the effects of a physical activity counselling approach aimed at reducing sedentary behaviour and building up to the public health guidelines (above). The physical activity facilitators work individually with patients over ten months to help patients develop a sense of competence, control and companionship through physical 
activity participation. This approach also helps to reduce the usual barriers of time, cost, inconvenience and lack of social support. This pragmatic intervention is likely to be implementable within the NHS, should it be effective.

\section{Summary}

In summary, while there is patient interest in the types of structured exercise programmes that the NICE guidelines recommend, based on a rather narrow evidence base, such programmes may create barriers for patients and GPs. There is no evidence that these types of programmes are cost-effective, with insufficient resources available to GPs having been identified across the country. ${ }^{19}$ It is early days yet, but physical activity counselling that entails a progressive approach using cognitive and behavioural strategies may well provide a

\section{REFERENCES}

I National Institute for Health and Clinical Excellence. Depression: the treatment and management of depression in adults. London: NICE; 2009.

2 Steptoe A, editor. Depression and physical illness. Cambridge: Cambridge University Press; 2006.

3 Badger F, Nolan P. Use of self-chosen therapies in depressed people in primary care. J Clin Nurs 2007; I6:1343-52. doi: I0.I I I I/ j.1365-2702.2007.01769.x

4 Taylor A. Increasing physical activity as a low intensity treatment for depression. In: Bennett-Levy J, Richards D, Farrand P et al., editors. Oxford Guide to low intensity CBT interventions. Oxford: Oxford University Press; 2010. p. I59-68.

5 Mead GE, Morley W, Campbell P et al. Exercise for depression. Cochrane Database Syst Rev 2009; 3:CD004366.

6 Scottish Intercollegiate Guidelines Network. Non-pharmaceutical management of depression in adults. Edinburgh: SIGN; 2010.

7 Morgan O. Approaches to increase physical activity: reviewing the evidence for exercise referral schemes. Public Health 2005 I 19:36 I-70. doi:I0.10 I6/j.puhe.2004.06.008

8 Williams $\mathrm{NH}$, Hendry M, France $\mathrm{B}$ et al. Effectiveness of exercise-referral schemes to promote physical activity in adults: systematic review. $\mathrm{Br} J$ Gen Pract 2007; 57:979-86. doi: I0.3399/0960I6407782604866

9 Craft LL, Perna FA, Freund KM et al. Psychosocial correlates of exercise in women with self-reported depressive symptoms. J Phys Act Health 2008; 5:469-80.

10 Seime RJ, Vickers KS. The challenges of treating depression with exercise: from evidence to practice. Clin Psychol Sci Pract 2006; | 3:194-7. doi:I0.1 I I I/j.| 468-2850.2006.00022.x

II Kirsch I, Deacon BJ, Huedo-Medina TB et al. Initial severity and antidepressant benefits; a meta-analysis of data submitted to the Food and Drug Administration. PLoS Med 2008; 5:e45. doi:I0.137I/ journal.pmed.0050045 new range of therapies that suit people who feel less suited to a structured exercise programme. Cognitivebehavioural therapies for depression rarely focus on increasing physical activity or, indeed, measure physical activity, but there is scope to explore new complex interventions approaches to promote exercise as a therapy. Such an approach also has the potential to fit within a stepped care system to treating depression, in which physical activity counselling is considered as a low-intensity treatment option within the increasing access to psychological therapies. ${ }^{4}$ Whether practitioners should or should not promote physical activity as a treatment for depression is likely to be decided by practitioners within a local context, informed by discussions on the pros and cons of the literature and the views of the patient.
12 Turner EH, Matthews AM, Linardatos E et al. Selective publication of antidepressant trials and its influence on apparent efficacy. N Engl J Med 2008; 358:252-60. doi:I0.1056/NEJMsa065779

13 Hill $A B$.The environment and disease: association or causation? Proc R Soc Med 1965; 58:295-300.

14 Taylor AH, Faulkner G. Inaugural editorial. Ment Health Phys Act 2008; I:I-8. doi:10.1016/j.mhpa.2007.II.00I

15 Dunn AL,Trivedi MH, Kampert JB et al. Exercise treatment for depression: efficacy and dose response. Am J Prev Med 2005; 28: I40-I. doi: I0.1016/j.amepre.2004.09.003

16 Dishman RK, O'Connor PJ. Lessons in exercise neurobiology: the case for endorphins. Ment Health Phys Act 2009;2:4-9. doi: I0.1016/j. mhpa.2009.01.002

17 Mental Health Foundation. Up and running! London: Mental Health Foundation; 2005. Available from: http://www.mentalhealth.org.uk/ campaigns/mhaw/exercise-and-depression/

18 Taylor $\mathrm{AH}$, Briscoe M, Faulkner AH. A web-based survey of mental health service users about the provision of physical activity as a therapy. Presented at the 3rd International Congress on Physical Activity and Public Health, 5-8 May 2010, Toronto, Canada.

19 Mental Health Foundation. Moving on up. London: Mental Health Foundation; 2009. Available from: http://www.mentalhealth.org.uk/ publications/

20 Taylor AH. The role of primary care in promoting physical activity. In: Riddoch C, McKenna J, editors. Perspectives in health and exercise. Basingstoke: Palgrave MacMillan; 2003. p. 153-80.

2I Department of Health. Exercise referral systems: a national quality assurance framework. London: $\mathrm{DOH} ; 200 \mathrm{I}$. Available from: http:// www.dh.gov.uk/assetRoot/04/07/90/09/04079009.pdf 